\title{
The prediction of perceptual-motor learning from independent verbal and motor measures'
}

DAVID L, KOHFELD

UNIVERSITY OF ILLINOIS

Forty male college students were administered motor and verbal pretests and were given learning trials on a criterion task which required both motor and verbal skills. The pretests were employed as predictors to determine the relationship of verbal and motor abilities to early and late stages of perceptual-motor performance. It is suggested that verbal comprehension is more important early in perceptual-motor learning while motor skill is more critical in later learning.

Several writers (James, 1890; Broadbent, 1958; Welford, 1958) have discussed the possibility that different components or abilities which contribute to learning early in practice may be different from those which facilitate later learning. Of particular interest has been the question of how so-called "verbal" and "motor" components interact with one another during the course of acquisition and later mastery of a task. Along experimental lines, Fleishman \& Hempel (1954) reported that non-motor factors, e.g., visualization, and spatial relations, contributed 46.1 per cent of the variance in the scores during the first stages of practice on a coordination test while motor factors, e.g., psychomotor coordination, and rate of movement contributed only 29.5 per cent. During the final stages of practice, non-motor factors accounted for 10.5 per cent while motor factors accounted for 74.5 per cent.

The study reported here involved the use of independent verbal and motor measures to predict Ss' scores on criterion task trials. The hypothesis under test was that the verbal predictor would best describe learning in early criterion task trials while the motor predictor would gain in predictive strength as learning progressed. Method

The Ss were 40 male undergraduates at the University of Illinois. The apparatus employed as the motor test was the Two-Hand Coordinator (the THC is described and pictured in Gazne \& Fleishman, 1959). All Ss were first given $151-$ min. trials on the THC with a $15 \mathrm{sec}$. intertrial interval. The score given each $\mathrm{S}$ was the mean time on target for all 15 trials. For the verbal test, a Memory Drum was used. The list of items put into the drum was ordered in a way that would correspond as closely as possible to the design of a 14-unit T-maze. To fulfill this requirement, $14 \mathrm{CVC}$ nonsense syllables were selected. Next the syllables ZEB and BEP were randomly paired with the 14 syllables, making the list as follows: KEV-ZEB, SOF-ZEB, BIM-BEP, LAZ-BEP, COR-BEP, etc. The Ss were given 15 trials with the same list, and were allowed a $15 \mathrm{sec}$, intertrial interval.
The score was the mean number of syllables and associates correctly anticipated for all 15 trials.

The construction of the criterion task involved a modification of the THC, and was designated the Modified THC. The motor, circular plate, and follower arm were removed from an intact THC. A 14 choice point Tmaze was cut into a $5 / 8$ in. plywood board which was, in turn, mounted and fastened on top of the THC. A $3 / 8$ in. diameter peg was mounted in the moving parts of the THC such that by alternating the movements of the handles, the peg could be made to traverse the alleys of the maze from start to finish. A wire was coiled around the peg and the end was attached to one pole of a 6-volt dry cell battery. Electrical contacts were wired to all the choice points and blind alleys of the maze and connected in such a way that when the moving peg touched any choice point, contact was made and a green light came on. Similarly, the choice of a blind alley gave a red light. The Ss were able to learn the maze only by means of the information gained from the red and green lights. Both lights were installed on a panel which entirely covered the maze; thus the $S$ was unable to see the peg or the maze as he operated the two handles. A total of 15 Modified THC trials were administered. A S's score on a trial was the time required to traverse the Modified THC from start to finish.

\section{Results}

The correlation between the mean time on target for the motor scores and mean correct for the verbal scores was .04 , not statistically significant. This result indicates that the verbal and motor measures tap independent ability traits, each of which accounts for separate and additive amounts of variance in the Modified THC trial scores. In order to examine the relationship of the motor and verbal predictors with the Modified THC trial scores, an analysis developed by Tucker (1960) was employed. The first step was to carry out a principal axis factor analysis on the Modified THC scores. Factor A accounted for 71 per cent of the variance in the matrix while factor $B$ accounted for 13 per cent. The remaining factors extracted each accounted for less than 5 per cent of the variance and thus were deleted from the analysis. The loadings of trials on principal factors $A$ and $B$ is shown in Fig. 1. Examination of Fig. 1 leads one to believe that differential loadings of trials on the two factors exists, since the trial loadings on factor $A$ increase sharply and then level beginning with trial 3 while those on factor B show a consistent decrease throughout. 


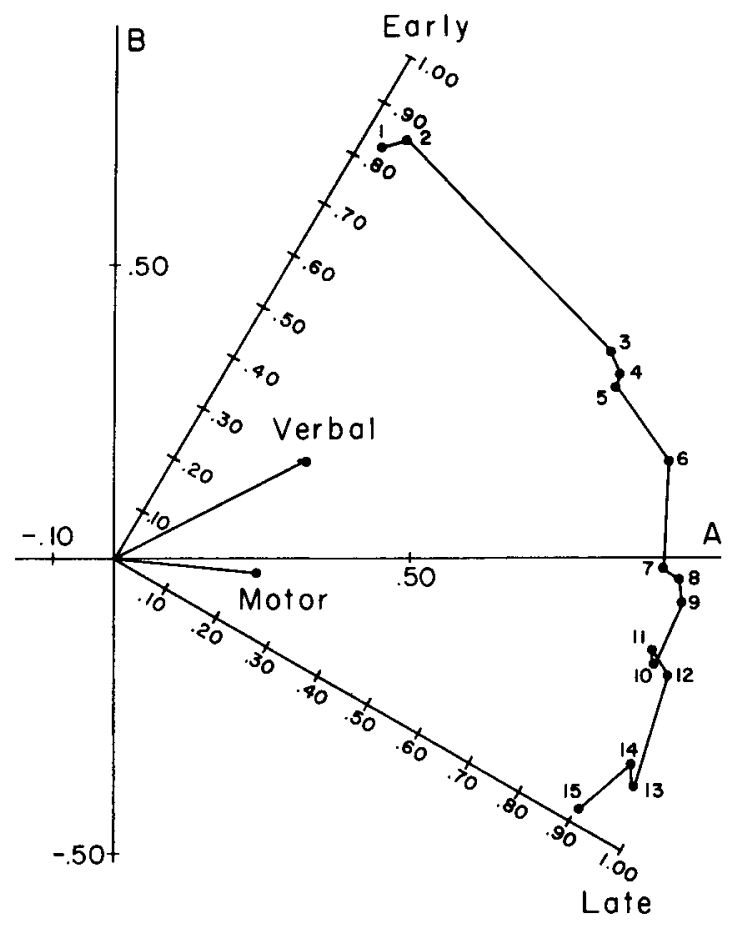

Fig. 1. Inter-factor plot of rotated factor loadings with projections of the motor and verbal predictors.

Figure 1 also shows the rotation made on factors $A$ and $B$. The new factors can now be appropriately identified as an Early and a Late factor. The "Early" and "Late," of course, refers to the fact that the early Modified THC trials load highest on the Early factor while the late trials load highest on the Late factor. The final step in the analysis was to obtain the correlation of the motor and verbal predictors with the rotated factors. The result showed that the motor predictor was correlated .23 with the Late factor and .12 with the Early factor whereas the verbal predictor yielded a correlation of .29 with the Early factor and one of .19 with the Late factor. The projection of the motor and verbal predictors onto the inter-factor plot of rotated factor loadings is presented in Fig. 1. With $\mathrm{df}=40$, a critical $\mathrm{r}$ of .257 is required for significance at the .05 level of confidence. The verbal predictor was significantly related to the Early factor but not to the Late factor. The motor predictor fell short of a significant relationship with the Late factor, but yielded a numerically higher correlation to the Late as opposed to the Early factor. While all the correlations presented are moderate in magnitude, the results depict a trend in the direction predicted by the research hypothesis.

\section{References}

Broadbent, D. E. Perception and communication. New York: Pergamon Press, 1958.

Fleishman, E. A., \& Hempel, W.E. Jr. Changes in factor structure of a complex psychomotor test as a function of practice. Psychometrika, 1954, 19, 239-252.

Gagne, R. M., \& Fleishman, E, A. Psychology and human performance. New York: Holt, 1959.

James, William. Principles of psychology (Vol. 1). New York: Holt, 1890 .

Tucker, L. R. Determination of generalized learning curves by factor analysis. Technical Report, 1960, Princeton University, Contract Nonr-1858 (15), Office of Naval Research.

Welford, A. T. Ageing and human skill. London: Oxford Univ. Press, 1958.

\section{Note}

1. This research was supported in part by the Office of Naval Research, Contract Nonr-1834 (39) to Dr. Ledyard $\mathbf{R}$ Tucker. The report is taken from an $M$. A. Thesis earned by the author at the University of Illinois, 1964. 